\title{
Optimized 0phthalmic: Advances in the Treatment of Ocular Diseases in Animals
}

\author{
KR Kurup, PV Parikh*, JK Mahla and DA Ratnu \\ Department of Veterinary Surgery and Radiology, Anand Agricultural University, India
}

Received: October 26, 2017; Published: November 06, 2017

*Corresponding author: Pineshkumar Parikh, Department of Veterinary Surgery and Radiology, College of Veterinary Science and Animal Husbandary, Anand Agricultural University (AAU), India; Email: parikhpv@gmail.com

Abbreviations: FDA: Food and Drug Administration; IOP: Intraocular Pressure; NSAIDS: Non Steroidal Anti-inflammatory Drugs; KCS: Kerato Conjunctivitis Sicca; AH: Aqueous Humor; CAIs: Carbonic Anhydrase inhibitors; PGAs: Prostaglandin F2 $\alpha$ Analogues

\section{Introduction}

The eye is a highly complex organ in terms of structure and function. It is a very sensitive organ, the function of which may be affected even with mild insult to its homeostasis, due to direct injury or due to other local or systemic diseases Scountzou [1]. The practice of ophthalmology, whether on humans or animals, can be reduced to the simple goal of getting the right pharmacological agent at the appropriate therapeutic dose to the target ocular tissue by a method that does not damage healthy tissue. In ocular disease, however, this simple goal becomes more challenging because of the highly sensitive ocular tissues (e.g., the uveal tract and retina) and the presence of tissue barriers to drug penetration, namely the lipophilic corneal epithelium, the hydrophilic corneal and sclera stroma, the conjunctival lymphatics, choroidal vasculature, and the blood-ocular barriers Weiner \& Gilge [2].

There are only a few veterinary ocular drugs that have been approved for marketing by the US Food and Drug Administration (FDA) (e.g., topical antibiotics [triple antibiotic], corticosteroids [neopolydex], cyclosporine [0.2\% Opt immune]). Most of the ocular medications veterinarians prescribe are those approved for human use, such as intraocular pressure (IOP) lowering medications, topical non steroidal anti-inflammatory drugs (NSAIDS), corticosteroids, etc. this approach has its limitations; it is wrong to assume that if a drug product works well in the human eye, it will work well in the animal eye and differences in eye anatomy and physiology of the animals encountered in veterinary practice, their unique diseases and specific responses to therapeutic agents are all sufficient reasons for encouraging and promoting research and development in veterinary ophthalmology Weiner [3].

\section{Drug Delivery System}

Potential routes of administration of drugs for ocular (adnexal, ocular surface, intraocular or orbital) diseases include topically applied ointments, solutions, or suspensions; systemic (parenteral or oral) administration, local (subconjunctival, intracameral, intravitreal, sub retinal, retro bulbar) injections or controlled drug delivery (hydro gels, inserts and prodrugs) Maggs [4]. In clinical practice the anterior segment of the eye (cornea, conjunctiva, sclera, anterior uvea) can be treated with topical ocular eye drops, the most commonly used dosage form in ocular drug treatment. Unfortunately, the ophthalmic topical formulations bear some drawbacks related to poor ocular bioavailability due to many anatomical and physiological barriers existing in the eye Gunda et al. [5]. Drugs are mainly eliminated from the precorneal lacrimal fluid by solution drainage, lacrimation and nonproductive absorption to the conjunctiva of the eye Lee \& Robinson [6]. These factors and the cornea1 barrier limit the penetration of the topically administered drug into the eye.

The normal commercial eye dropper delivers a drop volume of 25-56 $\mu$ l (average $39 \mu \mathrm{l}$ ) Lederer \& Harold [7]. When an eyedrop is instilled, the cul-de-sac may momentarily contain a $30 \mu \mathrm{l}$ volume, but the instilled solution is rapidly removed by spillage from the conjunctival sac or loss through the puncta to the lacrimal drainage system until the tears return to their normal volume. This rate decreases with viscosity and increases with larger eye drop volumes Lee \& Robinson [8]. Ocular administration of irritating drugs or vehicles increases the drug loss from the precorneal area to a further extent due to induced lacrimation Lee \& Robinson [6]. 
An important route of drug loss from the lacrimal fluid is systemic absorption through the conjunctiva of the eye. Due to the relative leakiness of the membrane, rich blood flow and large surface area, conjunctival uptake of a topically applied drug from the tear fluids is typically an order of magnitude greater than cornea1 uptake Urtti [9].

\section{Common Ocular Drugs}

\section{Antibacterial Drugs}

There are few veterinary-formulated ophthalmic antimicrobials available for topical application. A number of veterinary ophthalmic antimicrobial-corticosteroid combinations are also available. The most commonly available antibacterial drugs are "triple antibiotic" which contain neomycin, bacitracin, and polymixin, moxifloxacin, tobramycin, chloramphenicol, ciprofloxacin etc. One of the most common indications for administration of topical antibiotics in veterinary ophthalmology is corneal ulceration. One of the recent advancement is the use of cross-linked hyaluronan for the treatment of acute, trauma-induced, non- infected corneal ulcers in dogs and cats. REMEND ${ }^{\text {TM }}$ Corneal Repair Drops, with cross-linking technology, have been proven to heal corneal ulcers in as little as 48 hours.

Natural hyaluronan has been shown to promote migration of corneal epithelial cells and facilitate corneal healing. The crosslinked hyaluronan in Remend Corneal Repair Drops promotes cell migration and supports the animal's natural repair system by providing the optimal environment for healing. Not FDA approved for treatment of eye diseases in any animal as there has been no published clinical studies in dogs, cats and horses Yang et al. [10].
The oral and intravenous routes are seldom used in ophthalmology, because the blood ocular barrier impairs the achievement of satisfactory drug concentration in intraocular tissues. However tetracycline class of antibiotics like doxycycline is actively secreted by the lacrimal gland \& may reach therapeutic levels within the tear film after oral or parenteral administration and hence can be used for ocular infections Occhiutto et al. [11].

\section{Anti-inflammatory Agents}

Corticosteroids and nonsteroidal anti-inflammatory drugs (NSAIDs) have both been used topically and systemically to control adrenal, corneal, and intraocular inflammation Glaze [12]. Common commercially available topical anti-inflammatory drugs are dexamethasone, prednisolone, flurbiprofen, ketorolac, bromfenac, nepafenac etc. With numerous options for topical antiinflammatory therapy, it can be difficult for practitioners to know which medication to choose, and when. There are few concrete rules guiding use of ophthalmic anti-inflammatories in veterinary patients.

A. Rule 1: When a Corneal Ulceration is present, avoid topical corticosteroids.

B. Rule 2: Avoid topical NSAIDs in patients with glaucoma or predisposition to glaucoma.

C. Rule 3: Hydrocortisone is a very weak topical antiinflammatory agent.

D. Rule 4: When treating posterior segment disease, use systemic anti- inflammatory Giuliano [13].

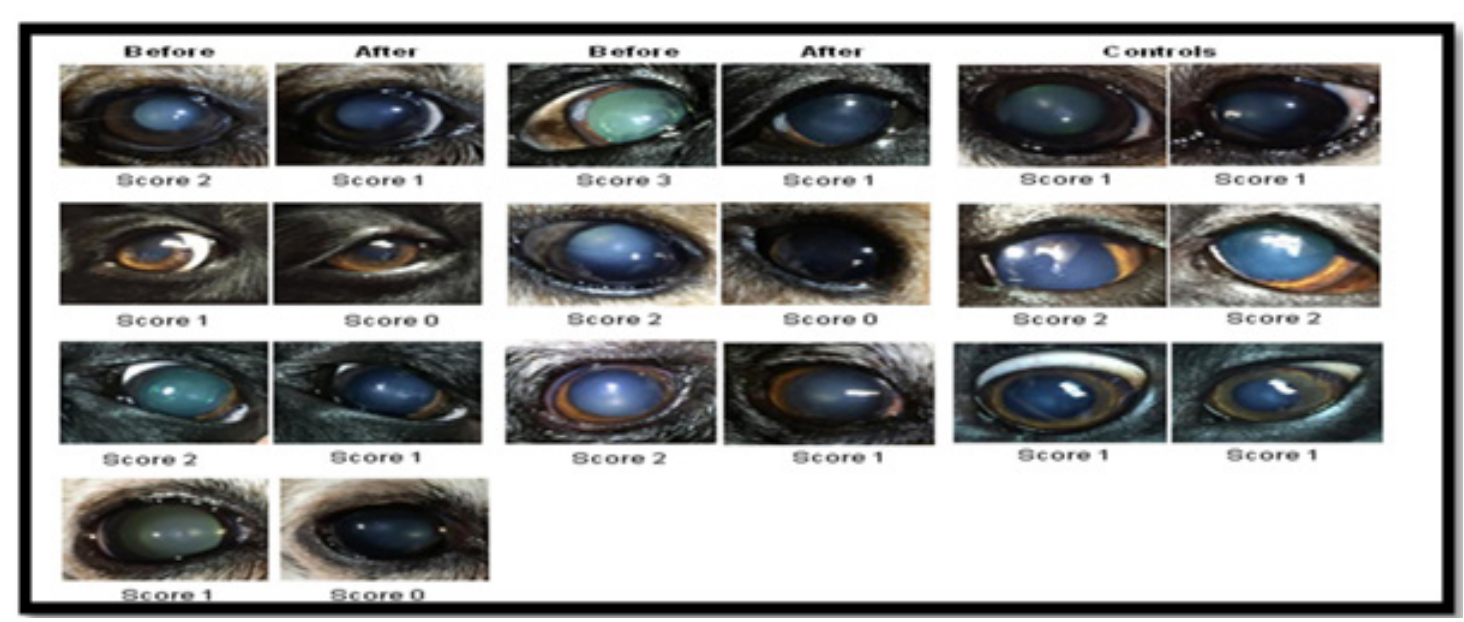

Figure 1: Anti-glaucoma Drugs.

Canine eyes with naturally occurring cataracts, before and after treatment.

Zhao et al. [14] came up with a steroid-based eye drop to dissolve the cataract right out of the eye's lens. this compound was tested in seven dogs including black labs, Queensland heelers and miniature pinschers with naturally occurring cataracts control group (two columns on right) show dog eyes that didn't receive treatment. However two columns on the left showed that after treatment for six weeks, there was an decrease in cloudiness of the cataract (Figure 1). Thus lanosterol proves to be revolutionary research in treatment of cataract. However, lanosterol needs several more years of study as all cataracts are not the same since the exact mechanism of how lanosterol manages to disperse the mass of protein remains unknown.

Glaucoma is one of the most frustrating and most difficult conditions to face in veterinary ophthalmology. The diagnosis and treatment can be quite challenging, and in most cases, despite 
intensive therapy, the disease process will progress and eventually lead to blindness and loss of the eye. Current pharmacological treatment of glaucoma focuses on indirect protection of the optic nerve, i.e. through lowering IOP. IOP can be decreased by surgical and medical (pharmacological) treatment. Medical treatment consists of topical and/or systemic application of ocular hypotensive drugs. The two principal ways to reduce IOP pharmacologically are by lowering aqueous humor $(\mathrm{AH})$ production and by increasing AH outflow. Although some ocular hypotensive drugs affect both pathways, they can be categorised by their dominant mechanisms of action as:

a) Those that inhibit the rate of $\mathrm{AH}$ production [Carbonic anhydrase inhibitors (CAIs), $\beta$-adrenergic antagonists and selective $\alpha 2$-adrenergic agonists]

b) Those that increase AH outflow [Nonselective adrenergic agonists, parasympathomimetics and prostaglandin F2 $\alpha$ analogues (PGAs)]

c) Those that dehydrate the intraocular space via the creation of an osmotic gradient (osmotic agents) Maslanka [15]. More recently, use of specific neuroprotection has been established for glaucoma. Such drugs are not expected to restore vision which has been lost prior to initiation of treatment but may very well help preserve vision Lipton [16]. Memantine, an NMDA channel blocker, has been shown to be neuroprotective in both rodent Schuettauf et al. [17] and monkey Hare et al. [18] models of glaucoma and is currently being investigated for canine glaucoma Ofri and Narfstrom [19].

\section{Mydriatics and Cycloplegics}

Mydriatic agents causes pupillary dilation either by paralysis of pupillary sphincter (eg.,cholinergic antagonists) or by stimulation of iris dilator muscle (eg. sympathomimetics). Cycloplegic agents act by causing paralysis of the ciliary muscle. Atropine causes pupillary dilatation accompanied by cycloplegia by blocking the actions of the short ciliary nerve which relaxes the sphincter muscle of the iris, dilating the pupils Kovalcuka et al. [20]. Tropicamide is a mydriatic of choice for intraocular diagnostic examinations because of rapid onset of action (20 min) and short duration of action (2-4 h) Taylor et al. [21].

\section{Tear Substitutes}

They are widely used in management of tear film disorders especially canine immune-mediated Keratoconjunctivitis Sicca (KCS) or dry eye which is a chronic ophthalmic disease resulting from deficiency of one or more elements in the precorneal tear film. They are commercially available as hydroxymethylcellulose (GENTEAL eyedrops) and carboxymethylcellulose (REFRESH eyedrops) Gelatt and Brooks [22].

\section{Calcineurin Inhibitors}

Both Cyclosporin A and tacrolimus are calcineurin inhibitors that reversibly inhibit T-cell proliferation. Tacrolimus is a macrolide antibiotic that has a similar immunopharmacological profile as CsA but is approximately 100 -times more potent than CsA. Formulations typically used are commercially available as $0.2 \%$ CsA ophthalmic ointment (Optimmune) and $1 \%, 2 \%$ or $3 \%$ componded CsA in olive or corn oil. They are used in the treatment of pigmentary keratopathy, chronic superficial keratitis (Pannus) and KCS Hendrix et al. [23].

\section{Antifungal Agents}

Antifungal agents are indicated in keratomycosis and ocular manifestation of canine systemic mycoses. Classes of antifungal agents used in veterinary ophthalmology include polyenes, azoles, allylamines, lipopeptides, and pyrimi-dines. The mode of action may be divided into those that target fungal cell membrane synthesis (azoles, allylamines, lipopeptides, and chitin synthase inhibitors) or function (polyenes and lipopeptides) and those that target nucleic acid synthesis (pyrimidines and SSD) Ford [24]. Antiviral agents are indicated in ocular manifestation of feline herpes virus. There are 2 topical antivirals that are commercially available at present namely Trifluridine and Vidarabine. A third, idoxuridine, is currently off the market, but is often compounded Stiles [25].

\section{Topical Ophthalmic Anesthetics}

It faciliate many veterinary ophthalmic diagnostic and therapeutic procedures such as tonometry, cytology of the cornea, removal of foreign body, intracameral and subconjunctival injections and surgical procedures. Currently, the two compounds most commonly used for topical ophthalmic anesthesia in veterinary ophthalmology are proparacaine $(0.5 \%)$ and tetracaine (1\%) and among these proparacaine is most widely used. Topical ophthalmic tetracaine is well tolerated in the horse as it produces maximal anesthetic effect and total duration of action in the horse following a single application of $0.5 \%$ tetracaine solution in 5.5 mins and 30 mins respectively. Hence, tetracaine is the preferred topical anesthetic in horses Monclin et al. [26].

\section{References}

1. Scountzou E (2003) Ophthalmic examination in dogs and cats. Veterinary Medicine Society 54(4): 329-334.

2. Weiner AL, Gilger BC (2010) Advancements in ocular drug delivery. Vet Ophthalmol 13(6): 395-406.

3. Weiner A (2006) Drug delivery systems in ophthalmic applications. In: T Yorio, A Clark, M Wax (Eds.), ( $1^{\text {st }}$ edn). Ocular therapeutics: Eye on new discoveries. Elsevier press/Academic press, New York, USA, p. 7-43.

4. Maggs DJ (2013) Ocular pharmacology and therapeutics. In: DJ Maggs, PE Miller, R Ofri (Eds.), $5^{\text {th }}$ edn. Slatter's Fundamentals of Veterinary Ophthalmology. St Louis, Missouri: Elsevier Saunders, USA, p. 27.

5. Gunda S, Hariharan S, Mandava N, Mitra, AK (2008) Barriers in ocular drug delivery. In: J Tombran-Tink \& CJ Barnstable (Eds.), $1^{\text {st }}$ edn. Ocular transporters in ophthalmic diseases and drug delivery. Totowa, NJ, USA: Humana press, pp. 399.

6. Lee VHL, Robinson JR (1979) Mechanistic and quantitative evaluation of precorneal pilocarpine disposition in albino rabbits. J Pharm Sci 68(6): 673-684.

7. Lederer CM, Harold RE (1986) Drop size of commercial glaucoma medications. Am J Ophthalmol 101(6): 691-694.

8. Lee VHL, Robinson JR (1996) Topical ocular drug delivery: Recent developments and future challenges. J Ocul Pharmacol 2(1): 67-108. 
9. Urtti A (2006) Challenges and obstacles of ocular pharmacokinetics and drug delivery. Adv Drug Deliv 58(11): 1131-1135.

10. Yang G, Espandar L, Mamalis N, Prestwich GD (2010) A cross-linked hyaluronan gel accelerates healing of corneal epithelial abrasion and alkali burn injuries in rabbits. Vet Ophthalmol 13(3): 144-150.

11. Occhiutto ML, Freitas FR, Maranhao RC, Costa VP (2012) Breakdown of the blood-ocular barrier as a strategy for the systemic use of nanosystems. Pharmaceutics 4(2): 252-275.

12. Glaze MB (1988) Contemporary pharmacology in veterinary ophthalmology. Semin Vet Med Surg (Small Animal) 3(1): 40-45.

13. Giuliano EA (2004) Nonsteroidal anti-inflammatory drugs in veterinary ophthalmology. Vet Clin Small Anim 34(3): 707-723.

14. Zhao L, Chen XJ, Zhu J, Xi YB, Hu LD, et al. (2015) Lanosterol reverses protein aggregation in cataracts. Nature 523(7562): 607-611.

15. Maslanka T (2015) A review of the pharmacology of carbonic anhydrase inhibitors for the treatment of glaucoma in dogs and cats. Vet J 203(3): 278-284.

16. Lipton SA (2003) Possible role for memantine in protecting retinal ganglion cells from glaucomatous damage. Surv Ophthalmol 48(1): 3846.

17. Schuettauf F, Quinto K, Naskar R, Zurakowski D (2002) Effects of antiglaucoma medications on ganglion cell survival: the DBA/2J mouse model. Vision Res 42(20): 2333-2337.

18. Hare WA, Wolde-Mussie E, Lai RK, Ton H, Ruiz G, et al. (2004) Efficacy and safety of memantine treatment for reduction of changes associated with experimental glaucoma in monkey, I: Functional measures. Invest Ophthalmol Vis Sci 45(8): 2625-2639.

19. Ofri R, Narfstrom K (2007) Light at the end of the tunnel? Advances in the understanding and treatment of glaucoma and inherited retinal degeneration. Vet J 174(1): 10-22.

20. Kovalcuka L, Birgele E, Bandere D, Williams DL (2015) Comparison of the effects of topical and systemic atropine sulfate on intraocular pressure and pupil diameter in the normal canine eye. Vet Ophthalmol 18(1): 43-49.

21. Taylor NR, Zele AJ, Vingrys AJ, Stanley RG (2007) Variation in intraocular pressure following application of tropicamide in three different dog breeds. Vet Ophthalmol 10(1): 8-11.

22. Gelatt KN, Brooks DE (2011) Clinical pharmacology and therapeutics. In: KN Gelatt \& J. P. Gelatt (Eds.), $1^{\text {st }}$ edn. Veterinary Ophthalmic Surgery. Philadelphia, USA: Elsevier Saunders. pp. 307-317.|

23. Hendrix DVH, Adkins EA, Ward DA, Stuffle J, Skorobohach B (2011). An Investigation Comparing the Efficacy of Topical Ocular Application of Tacrolimus and Cyclosporine in Dogs. Vet Med Int 12: 1-5.

24. Ford MM (2004) Antifungals and their use in veterinary ophthalmology. Vet Clin Small Anim 34(3): 669-691.

25. Stiles J (1995) Treatment of cats with ocular disease attributable to herpesvirus infection: 17 cases (1983-1993). J Am Vet Med Assoc 207(5): 599-603.

26. Monclin SJ, Farnir F, Grauwels M (2011) Determination of tear break-up time reference values and ocular tolerance of tetracaine hydrochloride eyedops in healthy horses. Equine Vet J 43(1): 74-77.

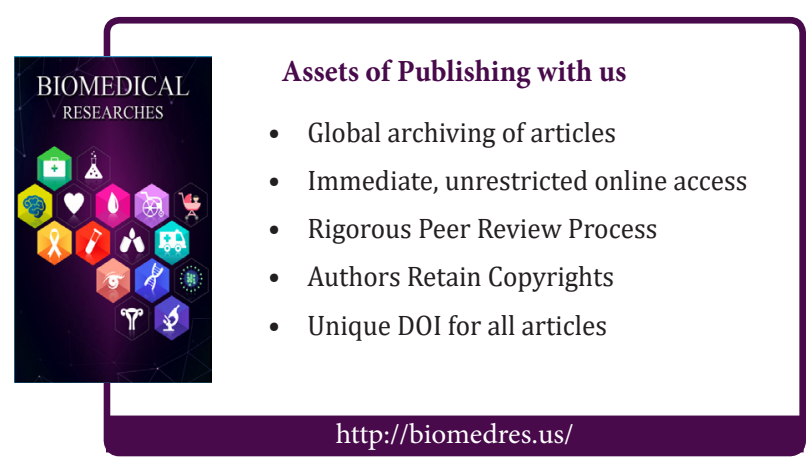

